\title{
Effect of antiretroviral drug (arved) on hepatic enzymes in albino rats
}

\section{${ }^{* 1}$ PETERS, DE; UWAKWE, AA; MONAGO, CC}

\author{
${ }^{I}$ Department of Biochemistry, College of Natural and Applied Sciences, Faculty of Chemical Sciences, \\ University of Port Harcourt, Rivers State. Nigeria
}

KEY WORDS: HIV, AIDs, Antiretroviral Drugs, Arved, Zidovudine, Lamivudine. Alkaline phosphate (ALP), Aspartate amino transferase (AST), Alanine amino transferase (ALT), hepatocellular necrosis.

\begin{abstract}
In the rush to put as many patients as possible on a potent ART, with very little or no laboratory monitory, limited attention has been given to side effects. This study was therefore designed to evaluate the effects of antiretroviral drugs arved ${ }^{\circledR}$, on aspartate amino transferase (AST), alanine amino transferase (ALT) and alkaline phosphatase (ALP) activities as well as histological effect on the liver tissue. A total of fifty two (52) albino rats were randomly divided into four groups labeled A, B, C and D and kept in a well ventilated room. All experimental groups shared the same environmental conditions. Group A served as the control and rats were treated with distil water. Rats in groups $\mathrm{B}, \mathrm{C}$ and $\mathrm{D}$ were, respectively treated with three different doses of arved $\left(1.07,3.21\right.$, and $\left.4.29 \mathrm{mg} \mathrm{kg}^{-1}\right)$, The drug was administered orally daily for $2,4,6$, and 8 consecutive weeks. Animals were sacrificed twenty four hours after the last treatment. Blood samples were collected into heparinized sample bottles for biochemical analysis. Result obtained in this study revealed that arved dose and time dependently, significantly $(\mathrm{P}<0.05)$ showed higher AST activity with the highest activity observed in treatment group D in wk8, (149.50 $\pm 1.91 \mathrm{IU} / \mathrm{L})$ when compared to the control group. The mean value of ALT activity for the drug in dose dependent manner was observed to be significantly $(\mathrm{P}<0.05)$ higher when compared to control value. The activity was highest in week 2 treatment group $\mathrm{D}(58.50 \pm 2.65)$. The drug dose dependently, produced significantly $(\mathrm{P}<0.05)$ higher ALP activity when compared to the control ALP activity. The highest activity was observed in week 2 treatment group D (214.50 \pm 14.75$)$ when compared to the control group A. The histopathological observation of liver tissues showed no morphological change in group D treated for 8 weeks as well as the control group. The rats had no morphologic evidence of hepatocellular necrosis or degeneration. In conclusion, treatment of HIV/AIDS patients with arved may likely result to liver damage possibly cholestatic liver injury at prolonged treatment. ( ) JASEM
\end{abstract}

\section{http://dx.doi.org/10.4314/jasem.v18 i2.28}

Introduction: Human Immunodeficiency Virus (HIV) is a lentivirus (a member of the retrovirus family) that causes Acquired Immunodefiency Syndrome (AIDS), (Weiss, 1993; Doueke et al., 2009). Sub-Saharan Africa has been known to be worst hit by the HIV/AIDS pandemic (Akinsete, 2002, Onwuliri et al 2003). In Nigeria, an estimated 3.6 percent of the population are living with HIV and AIDS (UNGASS 2010). Although HIV prevalence is much lower in Nigeria than in other African countries such as South Africa and Zambia, the size of Nigeria's population (around 162.5 million) means that by the end of 2009, there were an estimated 3.3 million people living with HIV ( UNAIDS 2010, UNDP 2011). Approximately 220,000 people died from AIDS in Nigeria in 2009 (UNAIDS 2010). With AIDS claiming so many lives, Nigeria's life expectancy has declined significantly. In 2010 the overall life expectancy was only 52 years (UNDP 2011). Firstline ART is now reaching $>3$ million people in low and middle income countries (WHO, 2008). Several dilemmas, including the balance between cost and toxicity, are major ongoing challenge in resourcelimited setting. Because of the limited availability, cost and convenience of fixed-dose combination, most patients receive first-line regimen, including nevirapine, lamivudine, zidovudine or stavudine. Arved is a nucleoside analogue reverse transcriptase inhibitor. Arved is a brand name for the domestically produced zidovudine-lamivudine preparation of antiretroviral drug called combivir. It is a fixed dose combination of two antiretroviral drugs, lamivudine (also called 3TC, with the brand name Epivir) and zidovudine (also called AZT, with the brand name retriovir). The combination of the two drugs has a stronger and more sustained effect than using either 
drug alone, and assist in reducing pill burden and in aiding compliance with the antiretroviral drug therapy. Both lamivudine and zidovudine are reverse transcriptase inhibitors which block the action of an enzyme, reverse transcriptase that the virus required for reproduction. It reduces the viral load in the body and raise $\mathrm{CD}^{+}$cell count. It was approved for use in the United States by the Food and Drug Administration (FDA) on September 26, 1997, making it the thirteenth approved antiretroviral.

In the rush to put as many patients as possible on a potent ART, limited attention has been given to side effects. Very little or laboratory monitoring is available. Thus, most available data relied on clinical observation and subjective report to assess adverse events the emergence of liver diseases as one of the major causes of death in people infected with HIV has paralleled the introduction of more effective antiretroviral therapies. Antiretroviral drug-related liver injury (ARLI) is defined by elevations in liver enzymes in serum, with alanine aminotransferase (ALT) characteristically greater than aspartate aminotransferase (AST). It is one of the greatest causes of treatment discontinuation in HIV-infected patients (Nunez et al., 2006). Its prevention and management is therefore very important among HIV infected patients who are to be placed on highly active antiretroviral therapy (HAART) (Palella et al., 2006). Surveys have reported an increased incidence of hepatic injury in HAART-treated patients and identified life threatening hepatotoxic events and end-stage liver disease in patients on antiretroviral ( Spengler et al., 2002). With the widespread use of HAART and the availability of new antiretroviral medications, ARLI has gained prominent attention owing to its negative impact on clinical outcomes. Drug associated hepatotoxicity also creates an economic burden on already strained medical budgets, since additional visits and hospital admissions are often required for appropriate patient care and management (Nunez et al., 2006). Furthermore, antiretroviral drug discontinuation hampers maintenance of HIV suppression. The severity of ARLI may range from the absence of symptoms to liver decompensation, and the outcome can range from spontaneous resolution to liver failure and death (Clark et al., 2002). Several highly active antiretroviral therapy (HAART) regimens are hepatotoxic and the liver is one of the vital organs useful in the metabolism of these drugs as well as in detoxification. It is therefore important that the liver which is the main biochemical hub of the body be monitored and those HAART regimens that may be toxic to it identified so that changes or modifications can be made to enhance patient care.

\footnotetext{
${ }^{*}$ PETERS, DE; UWAKWE, AA; MONAGO, CC
}

\section{MATERIALS AND METHODS}

Antiretroviral drug samples. Antiretroviral drug sample named arved used in this study was purchased from NAFDAC approved pharmaceutical store located opposite the gate of University of Port Harcourt Teaching Hospital, Alakahia, Rivers State of Nigeria. The details of the drug are stated below;

ARVED® -SNP 30,Active Ingredients (Zidovudine:300mg and Lamivudine:150mg) Manufactured by Cipla LTD., Plot No L-139 Verna,Goa 403722, India. For: Evans Medical PLC, Km. 32 Lagos Expressway, Lagos State. Batch. No: G973223

Specimen (Animal) Used For The Experiment: Fifty two (52) albino rats were purchased from the Department of Human Physiology, University of Nigeria Enugu Campus (UNEC) and acclimatized for one week at the Animal House of Biochemistry Department, University of Port Harcourt located at the botanical garden Choba Park. During acclimatization, the animals were fed with rat pellets and water ad libitum.

Chemicals And Reagents: All chemicals and reagents used in this study were of analytical grade. equipment.

Centrifuge (Universal 320, Hettic ZentrifugenGermany), refrigerator (Frestech), colorimeter (Jenway 6051 colorimeter; UK), weighing balance (Mettle Toledo. AB 204, Switzerland), Spectrophotometer (Beckman Coulter, DU 520 General Purpose UV / Visual), water bath (UNISCOPE-Sn801A surgifriend medicals, England).

Preparation Of Drug Solution For Administration: One tablet of the drug- arved was ground to a fine powder and dissolved in $100 \mathrm{ml}$ of distilled water to make a therapeutic dose concentration of $(4.29 \mathrm{mg} / \mathrm{kgBW})$ which served as group D drug treatment. Two other concentrations were prepared by adding $7.5 \mathrm{ml}$ and $2.5 \mathrm{ml}$ of distil water to $2.5 \mathrm{ml}$ and $7.5 \mathrm{ml}$ respectively of the therapeutic dose concentration to make dose concentrations of 3.21 and $1.07 \mathrm{mg} / \mathrm{kgBW}$ for groups $\mathrm{C}$ and B treatments.

Experimental Procedure: A total of fifty two albino rats (52) of weight range $(150-175 \mathrm{gBW})$ were randomly divided into four groups labelled A, B, C and D. Group A served as control and rats(n=4rats/dose) were treated with distilled water. Rats in groups $\mathrm{D}, \mathrm{C}$ and $\mathrm{B}$ ( $\mathrm{n}=4 \mathrm{rats} /$ dose) were orally treated with three different doses of arved (4.29, 3.21, 
$1.07 \mathrm{mg} / \mathrm{kgBW}$ ), daily for $2,4,6$ and 8 weeks. Animals were sacrificed twenty four (24) hours after the last treatment.

Drug Administration To Rats: $0.25 \mathrm{ml}$ of the drug solution was administered at the different concentrations to the animal in the groups using syringe. Administration was done orally twice daily.

Collection Of Blood And Preparation Of Serum: The rats were withdrawn from the cages in each of the group twenty four (24) hours after the last administration of drugs for 2,4,6 and 8 weeks and placed in a desiccator containing cotton wool soaked in chloroform to anaesthetize the rats. The blood samples were obtained by cutting the jugular vein of the rat on the neck by means of surgical blade and put in anticoagulant sample bottles smeared with lithiumheparin. The blood samples were spun at 5000rpm using MSE centrifuge to obtain serum. Rats were dissected and the liver was collected.

Measurement of AST (SGOT) and ALT (SGPT) The activities of glutamic-pyruvate transaminase and glutamic-oxaloacetate transaminase were analysed according to the method specified by Reitman and Frankel (1957).
Measurement of ALP.: Serum alkaline phosphatase activity was measured by the method of Rec (1972).

\section{Histopathological examination}

A small portion of the kidney tissues from groups A and $\mathrm{D}$ were excised immediately after sacrifice. Tissues were fixed in $10 \%$ formalin in phosphate buffer ( $\mathrm{pH} \mathrm{7.0)}$ ) for $24 \mathrm{hr}$ at room temperature for histopathology. Tissues were embedded in paraffin was and sections were cut 3-5 $\square \mathrm{m}$ slices and were stained with haematoxylin and eosin (H\&E) and observed under light microscope (Galigher et al., 1971).

Statistical Analysis: Results were analyzed using (SPSS) version 15 . The data were expressed using descriptive statistics and Analysis Of Variance (ANOVA). Multiple comparisons for the groups were done using Post Hoc Turkey (HSD) to test for the level of significance between means. A $p<0.05$ was considered to be statistically significant. Values are expressed in Means \pm Standard Deviation $(\mathrm{M} \pm \mathrm{SD})$. Superscript a: Indicates significant difference $(\mathrm{p}<0.05)$

\begin{tabular}{|c|c|c|c|c|}
\hline \multirow[b]{3}{*}{$\begin{array}{l}\text { Drug treatment/Control } \\
\text { groups }\end{array}$} & \multicolumn{4}{|c|}{ Weeks of Administration } \\
\hline & Week2 & Week4 & Week6 & Week8 \\
\hline & \multicolumn{4}{|c|}{$\mathrm{M} \pm \mathrm{SD}$} \\
\hline Group A (Control) & \multicolumn{4}{|c|}{$108.50 \pm 3.00$} \\
\hline Group B $(1.07 \mathrm{mg} / \mathrm{KgBW})$ & $115.25 \pm 3.77$ & $118.75 \pm 2.22$ & $119.67 \pm 1.25$ & $126.25 \pm 5.91^{\mathrm{a}}$ \\
\hline Group $\mathrm{C}(3.21 \mathrm{mg} / \mathrm{KgBW})$ & $124.25 \pm 4.35^{\mathrm{a}}$ & $127.00 \pm 2.58^{\mathrm{a}}$ & $126.25 \pm 1.71^{\mathrm{a}}$ & $136.67 \pm 6.94^{\mathrm{a}}$ \\
\hline Group D $(4.29 \mathrm{mg} / \mathrm{KgBW})$ & $142.5 \pm 15.95^{\mathrm{a}}$ & $140.50 \pm 1,70^{\mathrm{a}}$ & $131.00 \pm 2.94^{\mathrm{a}}$ & $149.50 \pm 1.91^{\mathrm{a}}$ \\
\hline
\end{tabular}

Result is represented in mean \pm standard deviation $(\mathrm{M} \pm \mathrm{SD})$

Superscript 'a' represents significant difference when compared to control value

Table 2 Effect of oral administration of antiretroviral drug(arved) on ALT(UI/L) level of albino rats for 8 weeks

\begin{tabular}{lcccc}
\hline & Week2 & Week4 & Weeks of Administration \\
Drug treatment/Control groups & & \multicolumn{4}{c}{ Week6 } & Week8 \\
Group A (Control) & & $30.00 \pm 2.45$ & $26.33 \pm 1.25$ & $37.50 \pm 1.29^{\mathrm{a}}$ \\
Group B $(1.07 \mathrm{mg} / \mathrm{KgBW})$ & $40.25 \pm 0.96^{\mathrm{a}}$ & $29.00 \pm 1.83$ & $30.50 \pm 1.29$ & $42.33 \pm 1.70^{\mathrm{a}}$ \\
Group C $(3.21 \mathrm{mg} / \mathrm{KgBW})$ & $48.00 \pm 2.16^{\mathrm{a}}$ & $30.25 \pm 1.71$ & $36.67 \pm 2.05^{\mathrm{a}}$ & $46.50 \pm 1.91^{\mathrm{a}}$ \\
\hline
\end{tabular}

Result is represented in mean \pm standard deviation $(\mathrm{M} \pm \mathrm{SD})$

Superscript 'a' represents significant difference when compared to control value

\begin{tabular}{|c|c|c|c|c|c|}
\hline \multirow{4}{*}{$\begin{array}{l}\text { Drug treatment/Control groups } \\
\text { Group A (Control) }\end{array}$} & \multicolumn{5}{|c|}{ Weeks of Administration } \\
\hline & Week2 & Week4 & & Week6 & Week8 \\
\hline & & & $\mathrm{M} \pm \mathrm{SD}$ & & \\
\hline & & & $140.75 \pm 11.15$ & & \\
\hline Group B(1.07mg/KgBW) & $173.25 \pm 7.63^{\mathrm{a}}$ & $151.00 \pm 14.09$ & & $156.33 \pm 2.05$ & $189.50 \pm 12.56^{\mathrm{a}}$ \\
\hline Group C $(3.21 \mathrm{mg} / \mathrm{KgBW})$ & $190.75 \pm 7.89^{\mathrm{a}}$ & $182.75 \pm 8.18^{\mathrm{a}}$ & & $163.75 \pm 4.35$ & $150.00 \pm 1.63$ \\
\hline Group $\mathrm{D}(4.29 \mathrm{mg} / \mathrm{KgBW})$ & $214.50 \pm 14.75^{\mathrm{a}}$ & $213.33 \pm 11.03^{\mathrm{a}}$ & & $200.00 \pm 2.83^{\mathrm{a}}$ & $187.50 \pm 7.59^{\mathrm{a}}$ \\
\hline
\end{tabular}

Result is represented in mean \pm standard deviation $(\mathrm{M} \pm \mathrm{SD})$

\footnotetext{
${ }^{*}$ PETERS, DE; UWAKWE, AA; MONAGO, CC
} 


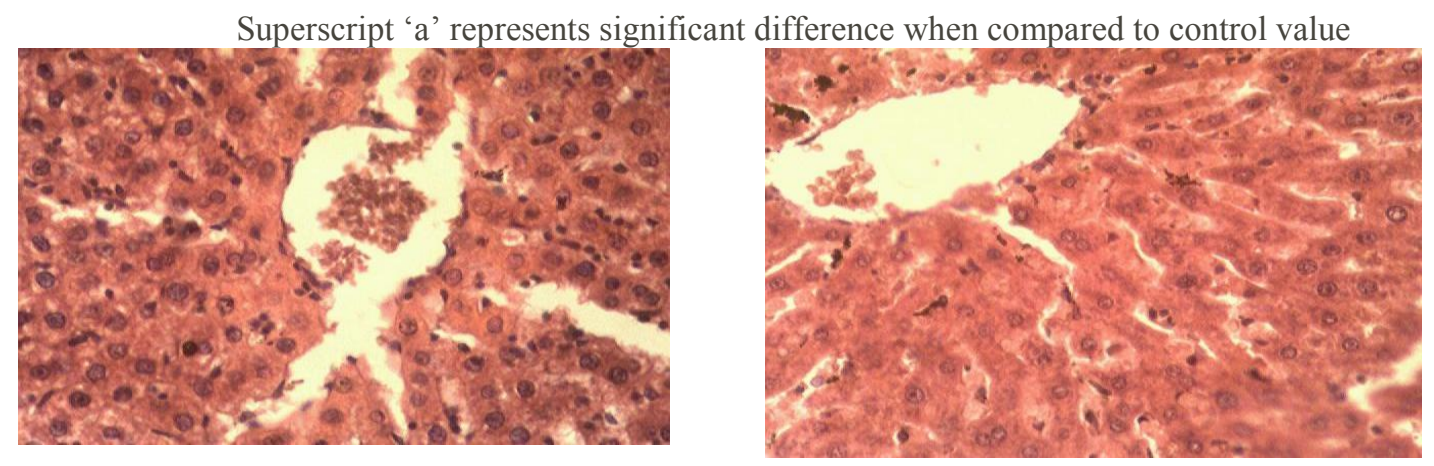

Plate 1: Photomicrograph of liver section of control group A (treated with distil water) showing normal hepatocyte

Plate 2: Photomicrograph of liver section treated $4.29 \mathrm{mg} / \mathrm{kgBW}$ (H\&Ex400)

of arved for weeks 8 (group D) showing normal liver architecture (H\&E x400)

\section{RESULT AND DISCUSSION}

The result from Table 1 revealed that mean serum aspartate amino transferase (AST) activity significantly increased $(\mathrm{p}<0.05)$ in the highest dose for arved treatment for all the weeks (range $131.00 \pm$ $2.94-149.50 \pm 1.91 \mathrm{U} / \mathrm{L})$ when compared to the mean value for the control $(108.50 \pm 3.00 \mathrm{U} / \mathrm{L})$. The highest level of AST activity was observed in week 8 group D arved $(149.50 \pm 1.91 \mathrm{U} / \mathrm{L})$. Alanine amino transferase (ALT) activity in Table 2 increased significantly $(\mathrm{p}<0.05)$ in all the treatment groups in week 8 with group D treatment having the highest activity $(46.50 \pm 1.91)$ when compared to the control value this trend was also observed in week 2 treatment. Alkaline phosphatase (ALP) activity in Table 3 followed similar pattern as (ALT) with significant increase in the group treated with the highest dose of arved for the entire period of treatment when compared to the mean value for the control. The highest activity for group D treatment was observed in week treatment and decreased as treatment progressed to week 8 in a time dependent manner. Similarly, week 2 treatment for the various groups also showed a dose depended pattern of significant increase. Histopathology observation of liver tissues from Plate 1 and 2 showed a normal liver architecture with normal hepatocytes, portal troads, central vein and sinusoids for control group and group D treated for 8 weeks respectively.

ALT is present in high concentrations in the liver and to a lesser extent in kidney, heart and skeletal muscle, pancreas, spleen and lung. Increase levels of ALT are generally a result of liver disease associated with some degree of hepatic necrosis such as cirrhosis, carcinoma, viral or toxic hepatitis and obstructive jaundice. Typically, ALT is generally higher than AST in acute viral or toxic hepatitis, whereas for most patients with chronic hepatic disease, ALT levels are generally lower than AST levels. Elevated ALT levels have also been found in extensive trauma and muscle disease, circulatory failure with shock, hypoxia, myocardial infarction and hemolytic disease (Penttila et al., 1975 and Allston, 1993). Elevated activities of these enzymes indicate cell damage which might have resulted from several mechanisms. These include metabolic host- mediated injury, hypersensitivity reaction, mitochondrion toxicity and immune reconstitution. All the nucleoside analogues have been implicated but hepatotoxicity may be more prevalent with stavudine, zalcitabine and didanosine. These three drugs have a higher affinity for mitochondrial polymerase- $\gamma$, and have a higher rate of toxicity than abacavir, zidovudine, lamivudine or tenofovir. This drug class (NRTIs) selectively inhibits DNA polymerases- $\gamma$ which is from the host cell and may affect nuclear or mitochondrial DNA. DNA polymerase- $\gamma$ implicated in the mitochondrion DNA (mtDNA) replication is targeted by NRTIs with subsequent mtDNA deletions and secondary deficits in mtDNA encoded enzymes of the mitochondrial respiratory chain. Oxidative phosphorylation impaired in mitochondrial with deficits in energy production (ATP), intracellular lipid accumulation upstream of the Krebs cycle and oxidative phosphorylation, and with production of lactate from anaerobic respiration. At the clinical level, the mitochondrial deficiency is responsible for adverse effects such as life-threatening lactic acidosis, hepatic steatosis and possible fat redistribution syndrome grouped under the entity of NRTI - induced mitochondrial cythopathy (kakuda, 2000; Cote et al., 2002; Vittecoo et al., 2002). The spectrum of mitochondrial toxicity of NRTI drugs ranges from nonspecific symptoms to lactic acidosis syndrome with fulminate hepatic failure (de Mendoza et al., 2004; Coghlan, et al., 2001). Zidovudine has been implicated in cases of fatal hepatic failure in advanced AIDS patients. These patients developed massive hepatomegaly and micro vesicular steatosis which progressed to fulminant hepatic failure (Frieman et al., 1993). Zidovudine can also cause 
acute hepatitis due to hypersensitivity, which resolved approximately 10 days after cessation of therapy. In these patients zidovudine was replaced with didanosine an zalcitabine without recurrence of toxicity (Wassef and Keiser, 1992 and Bissued et al., 1994). Lamivadine has been least reported to cause hepatotoxicity of all the NRTIs. A proposed reason is the low affinity of these drugs for mitochondrial DNA polymerase- $\gamma$. Transiently elevated levels of hepatic enzymes and bilirubin have been observed occasionally during treatment with lamivudine (Anthony, 2001). In a randomized trial (Mehta et al., 2006), lamivudine in combination with zidovudine and efavirez has better antiretroviral action with fewer side effects. However lamivudine is widely used and considered to be one of the best tolerated antiretroviral agents. The result in this study revealed approximately 2 fold $(\sim 2)$ significant increase in the activity of ALP. This result could possibly indicate cholestatic liver injury. ALP is a non specific enzyme which hydrolyses aliphatic, aromatic or heterocyclic phosphate compounds. ALP is produced by osteoblast of bone, intestinal mucosa. Mild increase in ALP was reported in children because of the increased osteoblastic activity and in pregnant women due to placental isoenzyme. Moderate increase in ALP level is seen in hepatic diseases such as infective hepatitis, alcoholic hepatitis or hepato cellular carcinoma. Very high activity of serum ALP than normal may be noticed in extrahepatic obstruction or cholestasis. ALP is also produced by epithelial cells of billiary canaliculi and obstruction of bile with consequent irritation of epithelial cells leads to secretion of ALP into serum. This variety of cell injury is the result of impaired bile flow and may be either intrahepatic or extrahepatic. In the case of extrahepatic cholestasis, both serum bilirubin and ALP are increased; the former because of a failure to excrete bilirubin, and the latter because of increased hepatic synthesis of ALP (Burke, 1975 and Kamath, 1996). When cholestasis is the result of focal intrahepatic disease, ALP is increased because of a cholestatic-induced increased synthesis, but bilirubin remains within normal limits. This is because as little as $10 \%$ of liver tissue is capable of maintaining normal serum bilirubin concentrations (Burke, 1975). This dissociation of serum ALP and bilirubin is characteristic of focal intrahepatic cholestasis (Kamath, 1996). However, the limitation in this study was the non inclusion of biluribin evaluation to make a precise conclusion. In liver enzyme assays earlier reported in this work, it was observed that mean serum AST, ALT and ALP activities significantly increased in the group D treatment without any noticeable morphological evidences of hepatocellular necrosis or degeneration. During liver injury, differences in the magnitude of biochemical signals and morphologic changes may arise because of differences in temporal onset of these effects. In addition, histopathology may underestimate the severity of injury when hepatobiliary markers are early indicators of injury, have short circulating halflives, or are depleted following an initial insult (Wang et al., 1997). Likewise, changes in hepatobiliary markers may be attributed to factors other than liver injury such as restraint (Landi et al., 1990; Valentine et al., 1990 and Nathwani et al., 2005), pharmacologically mediated effects on transporter function ( Zucker et al., 2001 and Campbell et al., 2004), or metabolism (Sapolsky et al., 2000; Jin et al., 2004 and Jackson et al., 2008). Most importantly in both animals and humans, relating biochemical changes to liver histopathology can be confounded by the complexity of druginduced liver injury because it often manifests as multiple histopathology findings rather than discrete or isolated changes (Travlos et al., 1996 and Tsui,2003), however, in this study, intrahepatic/extrahepatic cholestasis is suspected. In rats, the major circulating form of ALP is the intestinal rather than the liver isoenzyme ; however, total serum ALP activity is commonly measured. Total serum ALP activity is generally minimally increased in association with intrahepatic cholestasis in rat (Travlos et al., 1996) but can be markedly increased in rat bile duct ligation models. Intrahepatic cholestasis is a common manifestation of drug-induced liver injury in humans (Levy and Lindor, 2004; Lewis and Zimmerman, 1999) and is often the earliest manifestation of injury during liver transplant rejection (Lefkowitch, 2004). Similarly, transaminase increases are common in animal models of bile duct ligation (Aksu et al.,2009) and can be seen in patients with cholelithiasis and cholecystitis in which transaminase increases are in part attributed to local bile acid-mediated cytotoxicity (Chang et al., 2009; Nathwani et al., 2005). Although cholestasis can exert local effects, cholestasis is also mechanistically related to hepatocellular necrosis and apoptosis through a variety of processes including but not limited to Fas- and tumor necrosis factorrelated apoptosis-inducing ligand (TRAIL)-mediated activation of hepatocyte death receptors, bile acidmediated activation of mitochondrial pro-apoptotic pathways, and oxidative stress (Malhi et al.,2006). Biochemical evidence of cholestasis is based on the presence of more than twofold increases in Tbili, SBA, and/or ALP. The limitation of this study was non inclusion of Tbili and SBA assays, however ALP level significantly increased by approximately 2 -folds in this work. Cholestasis is primarily associated with phospholipidosis and/or biliary pathology, and less 
commonly with hypertrophy, lipidosis, glycogen accumulation, or parenchymal inflammation. In general, increases in ALT and AST with lesser increases or normal ALP favor cell necrosis, whereas the reverse points to cholestasis (Burke, 2002). The higher the ratio of ALT to ALP, the more likely the diagnosis is acute hepatic necrosis. There are exceptions: in acute alcoholic hepatitis, both ALT and AST (in particular ALT) are usually less than 10 times upper reference limits; and in ascending cholangitis caused by choledocholithiasis, AST and ALT may increase to a greater degree than ALP (Burke, 2002).These findings suggest that in the absence of hepatocellular necrosis, ALT, and ALP increases may be associated with cholestasis and can occur in the absence of histological changes related to hepatocellular or biliary injury.

In conclusion antiretroviral treatment with arved may be associated with hepatic enzymes induction in the short run, however hepatic function test should be monitored once every 2 weeks for the first two months after starting treatment regimen to determine onset of liver toxicity for patients with medical history of healthy liver at the commencement of treatment.

\section{REFERENCES}

Akinsete, I. (2002). HIV/AIDS: The Nigerian and Global situation analysis. National action committee on AIDS (NACA) Abuja, Nigeria.

Aksu, B., Umit, H., Kanter, M., Guzel, A., Inan, M., Civelek, S., Aktas, C. \& Uzun, H. (2009). Effects of sphingosylphosphorylcholine against cholestatic oxidative stress and liver damage in the common bile duct ligated rats. Journal of Pediatric Surgery. 44:702-710.

Allston, C.A. (1993). Non protein nitrogenous compounds and renal functions clinical chemistry concepts and applications, Anderson, S.C. Cockrayne, S. eds (W.B saunders Philadephia U.S.A) pp. 369.

Anthony, B. (2001). Hepatitis, HIV and your liver. AIDS Community. Research Initiative of America Community Forum Summary.

Bissued, F., Bruned, F. \& Haberselzer, F. (1994). Fulminant hepatitis with severe lactate acidosis in HIV-infected patients on didanosine therapy. Journal of Internal Medicine 235:367-371

Burke, M.D. (1975). Liver function. Human Pathology 6:273-86.

Campbell, S.D., de Morais, S.M., Xu, J.J. (2004). Inhibition of human organic anion transporting polypeptide OATP1B1 as a mechanism of drug-induced hyperbilirubinemia. Chemical and Biological Interaction. 150:179-187.

Chang, C.W., Chang, W.H., Lin, C.C., Chu, C.H., Wang, T.E. \& Shih, S.C. (2009). Acute transient hepatocellular injury in cholelithiasis and cholecystitis without evidence of choledocholithiasis. World Journal of Gastroenterology 15:3788-3792.

Clark, S., Creighton, S., Portmann, B., Taylor, C., Wendon, J. \& Cramp M. (2002). Acute liver failure associated with antiretroviral treatment for HIV: a report of six cases. Journal of Hepatology 36:295-301.

Coghlan, M., Sommadossi, J., Jhala, N., Many, W., Saag, M. \& Johnson, V. (2001). Symptomatic lactic acidosis in hospitalized antiretroviral-treated patients with HIV infection: a report of 12 cases. Clinical Infectious. Disease 33:1914-1921.

Cote, H.C., Brumme, Z.L. \& Craib, K.J. (2002). Changes in mitochondrial DNA as a maker of nucleoside toxicity in HIV-infected patients. New England Journal of Medicine 346:811-820.

de Mendoza, C., de Ronde, A., Smolders, K., Blanco, F., GarciaBenayas, T. \& de Baar, M. (2004). Changes in mitochondrial DNA copy number in blood cells from HIV-infected patients undergoing antiretroviral therapy. AIDS Research in Human Retroviruses. 20:271-273.

Doueke, D.C., Roederer, M. \& Koup, R.A. (2009). Emerging concepts in the immunopathogenesis of AIDS". Annual Review in Medicine 60: 471-84.

Freiman, J., Helfert, K., Hamrell, M. \& Stein, D. (1993). Hepatomegaly with severe steatosis in HIV-seropositive patients. AIDS 7:379-385.

Galigher et al. 1971. Essentials of Practical Microtechnique, 2nd Ed. Lea and Febiger, Philadelphia. pp -77.

Jackson, E.R., Kilroy, C., Joslin, D.L., Schomaker, S.J., Pruimboom-Brees, I. \& Amacher, D.E. (2008). The early effects of short-term dexamethasone administration on hepatic and serum alanine aminotransferase in the rat. Drug and Chemical Toxicology 31:427-445.

Jin, J.Y., DuBois, D.C., Almon, R.R. \& Jusko, W.J. (2004). Receptor/gene-mediated pharmacodynamic effects of methylprednisolone on phosphoenolpyruvate carboxykinase regulation in rat liver. Journal of. Pharmacological and Experimental Therapeutics. 309:328-339.

Kakuda, T.N. (2000). Phamacology of nucleoside and nonnucleoside reverse transcriptase inhibitor-induced mitochondrial toxicity. Clinical Therapeutics 22:685-708

Kamath, P.S. (1996). Clinical approach to the patient with abnormal liver test results. Mayo Clinical Proceedings 71:1089-95.

Landi, M., Kissinger, J.T., Campbell, S.A., Kenney, C.A. \& Jenkins, E.L.(1990). The effects of four types of restraint on serum alanine aminotransferase and aspartate aminotransferase in the Macaca fascicularis. Journal of American Journal of Toxicology. 9:517-523.

Lefkowitch, J.H. (2004). Histological assessment of cholestasis. Clinical Liver Disease 8:27-40.

Levy, C. \& Lindor, K.D. (2004). Drug-induced cholestasis. Clinical Liver Disease 7:311-320. 
Lewis, J.H. \& Zimmerman, H.J. (1999). Drug- and chemicalinduced cholestasis. Clinical Liver Disease 3:433-464.

Malhi, H., Gores, G.J., Lemasters, J.J. (2006) Apoptosis and necrosis in the liver: a tale of two deaths? Hepatology 43:S31-S44.

Mehta, S.H., Astemborski, J., Sterling, T.R., Thomas, D.L. \& Vlahov, D. (2006). Serum Albumin as a Prognostic Indicator for HIV Disease Progression. AIDS Research in Human Retroviruses. 22:14 -21

Nathwani, R.A., Pais, S., Reynolds, T.B. \& Kaplowitz, N. (2005). Serum alanine transferase in skeletal muscle diseases. Hepatology 41:380-382.

Nunez, M.J., Martin-Carbonero, L. Moreno, V., Valencia, E., Garcia-Samaniego, J., and Gonzalez- Castillo, J. (2006). Impact of antiretroviral. AIDS 22: 825-829.

Onwuliri, V.A., P. Kanki, M.M. Umeh and A. Awari, 2003. Educating sex workers in Nigeria. $13^{\text {th }}$ International Conf. AIDS STDS Africa, Nairobi, Sept. 21st - 26th 2003 No. 126598 pp: 6.

Palella, F., Baker, R., Moorman, A., Chmiel, J., Wood, K. and Brooks, J. (2006). Mortality in the highly active antiretroviral therapy era: changing causes of death and disease in the HIV outpatient study. Journal of Acquired Immune Deficiency Syndrome 43: 27-34.

Penttila, I.M., Jokela, H.A., Viitala, A.J., Heikkinen, E., Nummis. \& Pystynen, P. (1975). Activities of aspartate and alanine animotransterases and alkaline phosphatase in sera of healthy subjects. Scand. Journal of Clinical Laboratory Investigation 35: 275-284.

Rec, G.S.C.C. (1972). Colorimetric method for serum alkaline phosphatase determination. Journal of Clinical Biochemistry 10(2): 182 .

Reitman, S. \& Frankel, S. (1957). A colourimetric method for the determination of serum glutamic-oxaloacetic and glutamicpyruvic transaminase. American Journal Clinical Pathology 28: 56-61.

Sapolsky, R.M., Romero, L.M. \& Munck, A.U. (2000) How do glucocorticoids influence stress responses? Integrating permissive, suppressive, stimulatory, and preparative actions. Endocrime Review 21:55-89.

Spengler, U., Lichterfeld, M. \& Rockstroh, J.K. (2002). Antiretroviral drug toxicity-Therapy. ReutersHealth
Travlos, G.S., Morris, R.W., Elwell, M.R., Duke, A., Rosenblum, S. \& Thompson, M.B. (1996). Frequency and relationships of clinical chemistry and liver and kidney histopathology findings in 13-week toxicity studies in rats. Toxicology 107:17-29.

Tsui, W.M.S. (2003). Drug-associated changes in the liver. Current Diagnostic Pathology. 9:96-104.

UNAIDS (2010). 'UNAIDS report on the global AIDS epidemic'

UNDP (2011). 'Human Development Report 2011'

\section{UNGASS (2010). 'UNGASS Country Progress Report: Nigeria'}

Valentine, B.A., Blue, J.T., Shelley, S.M., Cooper, B.J. (1990). Increased alanine aminotransferase activity associated with muscle necrosis in the dog. Journal of Vetenary Internal Medicine. 4:140-143.

Vitecoo. D., Jardel, C. \& Barthelemy, C. (2002). Mitochondrial damage associated with long-term antiretroviral treatment: Associated alteration or causal disorder? Journal of Acquired Immune Deficiency Syndromes 31:299-308.

Wang, P-Y., Kaneko, T., Tsukada, H., Nakano, M., Nakajima, T. \& Sato, A. (1997). Time courses of hepatic injuries induced by chloroform and by carbon tetrachloride: comparison of biochemical and histopathological changes. Arch. Toxicology 71:638-645.

Wasseef, M. \& Kelser, P. (1992). Hypersensivity to zidovudine: Report of a case and review of the literature. American Journal of Medicine 93:94-96

Weiss, R.A. (1993). How does HIV cause AIDS? Science 260 (5112): 1273-9.

World Health Organisation (2008). Towards Universal Access: scaling up priority HIV/AIDS interventions in the health sector: progress report. Available from http://www.who.int/hiv/mediacenter/2008/progresssreport/en /index.html

Zucker, S.D., Qin, X., Rouster, S.D., Yu, F., Green, R.M., Keshavan, P., Feinberg, J. \& Sherman, K.E. (2001). Mechanism of indinavir-induced hyperbilirubinemia. Proceedings of the National Academy of Sciences U.S.A. 98:12671-12676. 